Audiology

Neurotology
Audiol Neurotol 2010;15:44-56

DOI: $\underline{10.1159 / 000219487}$
Received: September 28, 2007

Accepted after revision: January 26, 2009

Published online: May 21, 2009

\title{
Speech Perception Benefit for Children with a Cochlear Implant and a Hearing Aid in Opposite Ears and Children with Bilateral Cochlear Implants
}

\author{
Mansze Mok ${ }^{a}$ Karyn L. Galvin ${ }^{a}$ Richard C. Dowell ${ }^{a}$ b Colette M. McKay ${ }^{c}$ \\ ${ }^{\mathrm{a}}$ Department of Otolaryngology, University of Melbourne, and ${ }^{\mathrm{b}}$ Cochlear Implant Clinic, Royal Victorian Eye \\ and Ear Hospital, East Melbourne, Vic., Australia; 'School of Psychological Sciences, University of Manchester, \\ Manchester, UK
}

\section{Key Words}

Binaural hearing $\cdot$ Cochlear implant $\cdot$ Hearing aids ·

Speech perception

\begin{abstract}
The aims of the present study are to investigate: (1) the effect of using a hearing aid (HA) or a second cochlear implant (2nd $\mathrm{Cl}$ ) on speech recognition in noise for children; (2) the ability to perceive phoneme groups of different frequencies when using a $\mathrm{Cl}$ and an $\mathrm{HA}$ in opposite ears (bimodal fitting) and when using a $\mathrm{Cl}$ in each ear (bilateral implant fitting), and (3) the relationship between aided thresholds in the HA ear and bimodal advantage. Thirteen school-age children who consistently used a bimodal or bilateral implant fitting participated. Perception was evaluated using consonant-nucleusconsonant words presented from in front with noise from either side. Significant bimodal or bilateral $\mathrm{Cl}$ advantage in speech perception was demonstrated by most subjects in at least 1 noise condition. Comparisons indicated that the bimodal advantage obtained by the bimodal subjects was greater than the bilateral $\mathrm{Cl}$ advantage obtained by the bilateral-implant subjects in the noise front condition, but also suggested that the $2 \mathrm{nd} \mathrm{Cl}$ may provide more functional advantage in real life. The mechanisms underlying the advantage provided by the second device appear to be different
\end{abstract}

in the bimodal and bilateral groups. Information transmission analysis did not show a clear difference between the groups in the pattern of advantage across phoneme groups. For the bimodal subjects, those with better aided thresholds at low frequencies and poorer aided thresholds at $4 \mathrm{kHz}$ demonstrated greater bimodal advantage. Overall, these findings encourage the use of bimodal and bilateral implant fittings for children, provide insight into the individual variability in bimodal outcome, and enhance understanding of the differences between an $\mathrm{HA}$ and a $2 \mathrm{nd} \mathrm{Cl}$ when used together with an implant in the opposite ear.

Copyright $\odot 2009$ S. Karger AG, Basel

\section{Introduction}

In recent years there has been an increasing number of cochlear implant (CI) users wearing a hearing aid (HA) or a second (2nd CI) in the opposite ear. Such use of a second device in the opposite ear is often referred to as a bimodal fitting or bilateral implant fitting. The potential advantages of a bimodal or bilateral implant fitting over a single CI include improved speech perception in background noise, improved awareness of soft sounds, improved sound localization, and improved sound quality [see for example, van Hoesel and Tyler, 2003; Ching et al.,

\section{KARGER}

Fax +4161306 1234 E-Mail karger@karger.ch www.karger.com
(C) 2009 S. Karger AG, Basel

1420-3030/10/0151-0044\$26.00/0

Accessible online at:

www.karger.com/aud
Karyn Galvin

Audiology, Hearing and Speech Sciences

The University of Melbourne, 550 Swanston Street

Melbourne, Vic. 3010 (Australia)

Tel. +61 39035 5323, Fax +61 39347 8047, E-Mail kgalvin@unimelb.edu.au 
2004; Dunn et al., 2005; Kong et al., 2005; Litovsky et al., 2006b; Mok et al., 2006; Galvin et al., 2007]. For schoolage children, the potential to improve speech perception in background noise is of particular importance, as they are often required to listen in noisy environments in educational settings. The present study focuses on speech perception benefit in noise for school-age children with a bimodal or bilateral implant fitting.

Several studies have reported speech perception benefit for children using a bimodal or bilateral implant fitting compared to a single CI, though little is known about the underlying mechanisms for such benefit. For children with a bimodal fitting, some of the benefit may arise from having the two different types of devices. CIs can provide greater high-frequency information than HAs, whilst HAs can provide finer low-frequency spectral information through acoustic stimulation. It is possible that the auditory system can integrate the complementary signals from the two different devices, resulting in improved speech perception. Results of a study on adults using a bimodal fitting suggested that bimodal advantage for speech perception could be due to the improved perception of the low-frequency components of speech [Mok et al., 2006]. However, whether the same mechanism occurs for children using a bimodal fitting has not been investigated. In contrast to adults with a post-lingual hearing loss, most children with hearing loss lack the experience of normal binaural hearing in the early years of life. Due to differences in the development of the binaural auditory system, results obtained for adults cannot simply be assumed to apply to children. For children with bilateral implants, the benefit could arise from having both devices providing speech information across a wide range of frequencies. Hence, through binaural loudness summation and binaural redundancy, having the 2nd CI may improve perception of a wide range of phonemes, in particular, the soft, high-frequency consonants, which are often difficult to perceive. This, however, has not been investigated in adults or children with bilateral implants. Using information transmission analyses, it is possible to determine the frequencies of phoneme groups that contribute to the advantage provided by a second device, be it an HA or a 2 nd CI.

Previous research (as noted above) on the additional benefit provided by bimodal and bilateral implant fittings has shown great variability among individuals, but has not confirmed the factors contributing to this variability. Of particular interest to this study is the relationship between useable residual hearing with the HA ear and bimodal advantage. Useable residual hearing may be represented by unaided or aided hearing thresholds. Aided thresholds provide more information than unaided thresholds because they are a measure that incorporates the impact of the device (in this case, the HA) on perceptual performance. Interestingly, a study of children with bimodal fittings showed no correlation between the degree of hearing loss in the non-implanted ear and bimodal advantage [Ching et al., 2004]. However, a study of adults with bimodal fittings showed that those with poorer aided thresholds at mid-to-high frequencies $(1,2$, and $4 \mathrm{kHz}$ ) demonstrated greater bimodal advantage [Mok et al., 2006]. This suggests that the mid- to high-frequency information from the HA potentially conflicts with the information from the CI. It is possible that aided thresholds, rather than the degree of loss, are related to bimodal advantage, given that gain adjustments of the HA have been shown to affect bimodal advantage [Ching et al., 2001]. As no previous study has investigated the relationship between aided thresholds in the non-implanted ear and bimodal advantage in children, this is examined in the present study.

With the increased interest in bilateral implants in recent years, the question of whether an individual would gain more benefit from a contralateral HA or a 2 nd CI has become very important for establishing clinical selection criteria for a 2 nd CI. For an individual with no residual hearing for HA use in the non-implanted ear, the decision may be more straightforward as many studies have reported additional benefit from bilateral implants compared to a single CI [e.g. van Hoesel and Tyler, 2003; Galvin et al., 2007]. However, some families do hold other concerns regarding, for example, appearance or the child's potential to benefit from future technologies [Galvin et al., in press]. For an individual with access to the speech spectrum in the non-implanted ear, the decision involves weighing the benefit of an HA against that of a 2nd CI. Currently, differences between the speech perception benefit provided by an HA versus a 2nd CI are poorly understood. Comparisons of results with bimodal and bilateral implant fittings across different studies are difficult due to the differences in test methodologies and age groups. So far, only two studies have made direct, comparisons [Litovsky et al., 2006a; Mok et al., 2007]. Litovsky et al. [2006a] compared speech recognition in noise for children with bimodal or bilateral implant fittings using a closed-set spondee test. However, no statistically significant advantage from using an $\mathrm{HA}$ or a 2 nd CI was reported, even with the noise on the side of the implant or first implant. This could be due to the large variability in the individual results and/or the type of test 
material used. Mok et al. [2007] compared speech detection in noise performance for children with bimodal or bilateral implant fittings. Results showed that, when the noise was presented from the front, the children with the bimodal fitting gained greater advantage from the HA than the children with the bilateral implants gained from the 2 nd CI. In contrast, when the noise was presented from the side of the implant or first implant, the children with bilateral implants gained greater advantage. However, given that the underlying mechanisms of detection and recognition are potentially different, whether the same pattern of benefit occurs for speech recognition in noise remains unclear. A deeper understanding of the differences between bimodal and bilateral implant fittings will allow families to make a more informed choice between an HA and a 2nd CI for children who have significant residual hearing in the non-implanted ear. The present study compares speech recognition benefit and types of additional speech information gained from an $\mathrm{HA}$ versus a 2 nd $\mathrm{CI}$ using an open-set speech recognition test.

The aims of the present study were: (1) to investigate the effect of using an HA or a 2nd CI on speech recognition in noise for children; (2) to investigate the ability to perceive phoneme groups of different frequencies when using bimodal or bilateral implant fittings compared to a single CI, and (3) to explore the relationship between aided thresholds in the HA ear and bimodal advantage.

\section{Method}

Subjects

A total of 13 subjects participated in the study, including subjects who used a CI and an HA in opposite ears (bimodal subjects, $\mathrm{n}=9$ ), and subjects who used bilateral CIs (bilateral subjects, $\mathrm{n}=4)$.

Bimodal Subjects. All 9 bimodal subjects (Bim1-Bim9) had a bilateral severe-profound sensorineural hearing loss. The hearing loss was assumed to be congenital for all subjects, though subject Bim6 had a congenital moderate hearing impairment that deteriorated to a profound loss by 6 years of age. The mean age at cochlear implantation was 6.5 years $(\mathrm{SD}=2.7)$, and all subjects had been full-time users of a CI and an HA in opposite ears since implantation. A normal type A tympanogram (middle ear pressure $>-100 \mathrm{daPa}$ ) was obtained in the non-implanted ear at each test session. The mean age at testing was 11.8 years $(\mathrm{SD}=2.1)$. Details of the subjects' hearing loss, CI and HA are provided in table 1.

For all 9 subjects, bilateral HAs were worn consistently prior to cochlear implantation. Aided thresholds in the non-implanted ear are provided in table 2 (obtained with the adjusted volume settings after the loudness-balancing procedure outlined below). All subjects had access to part of the long-term average speech spectrum of $70 \mathrm{~dB}$ SPL with their current HA. The HAs were fitted according to the NAL-RP fitting strategy. The aided thresholds were slightly lower than the required aided thresholds calculated from the NAL-RP prescription by $3.8 \mathrm{~dB}(\mathrm{SD}=9.1)$ when averaged across subjects and the frequencies of $250 \mathrm{~Hz}, 500 \mathrm{~Hz}$, and $1 \mathrm{kHz}$. Subjects Bim2, Bim3, Bim5, and Bim6 used an HA with linear gain and no noise suppression. Subjects Bim1, Bim4, Bim7, Bim8, and Bim9 used an HA with input compression and noise reduction algorithms. The compression ratios and knee points varied from 1.3:1 to $2: 1$ and from 61 to $75 \mathrm{~dB}$ SPL, respectively. Only subject Bim9 normally wore an HA with a direction-

Table 1. Details of age, hearing loss, CI and HA for bimodal subjects

\begin{tabular}{|c|c|c|c|c|c|c|c|c|}
\hline Subject & $\begin{array}{l}\text { Age } \\
\text { years }\end{array}$ & Etiology & $\begin{array}{l}\text { Age at bilat- } \\
\text { eral HA fit- } \\
\text { ting, months }\end{array}$ & HA type & $\begin{array}{l}\text { Age at CI } \\
\text { implanta- } \\
\text { tion, years }\end{array}$ & $\begin{array}{l}\text { CI } \\
\text { usage } \\
\text { years }\end{array}$ & CI type & $\begin{array}{l}\text { CI } \\
\text { Strategy }\end{array}$ \\
\hline Bim1 & 11.4 & unknown & 10 & Siemens Music D SP & 5.4 & 6.0 & CI24M & ACE \\
\hline $\operatorname{Bim} 2$ & 9.2 & unknown & 6 & Bernafon Audioflex 120 Super Power & 3.9 & 5.3 & CI24M & $\mathrm{ACE}$ \\
\hline Bim3 & 14.9 & unknown & 21 & Bernafon Audioflex 120 Super Power & 11.0 & 3.9 & $\mathrm{CI} 24 \mathrm{R}(\mathrm{CS})$ & $\mathrm{ACE}$ \\
\hline Bim4 & 12.4 & unknown & 12 & Siemens Music Pro SP & 6.4 & 6.0 & CI24M & SPEAK \\
\hline Bim5 & 12.4 & unknown & 18 & Bernafon Audioflex 120 Super Power & 5.8 & 6.6 & CI24M & SPEAK \\
\hline Bim6 & 14.2 & CMV & 3 & Bernafon Audioflex 120 Super Power & 10.2 & 4.0 & CI24R(CS) & ACE \\
\hline $\operatorname{Bim} 7$ & 12.0 & unknown & 18 & Siemens Music Pro SP & 9.2 & 2.8 & CI24R(CS) & $\mathrm{ACE}$ \\
\hline Bim8 & 9.3 & $\begin{array}{l}\text { Pendred's } \\
\text { syndrome }\end{array}$ & 4 & Siemens Music Pro SP & 4.9 & 4.4 & CI24R(CS) & $\mathrm{ACE}$ \\
\hline Bim9 & 12.8 & unknown & 20 & Siemens Triano SP & 4.6 & 8.2 & $\mathrm{CI} 24 \mathrm{M}$ & $\mathrm{ACE}$ \\
\hline
\end{tabular}

CI24M = Straight-array implant with 22 electrodes plus 2 extracochlear electrodes; ACE = advanced combination encoder; CI24R(CS) = curved-array implant with 22 electrodes plus 2 extracochlear electrodes; SPEAK = spectral peak speech coding strategy; $\mathrm{CMV}=$ cytomegalovirus. 
al microphone, and this was switched to omnidirectional for all testing. All subjects were using an ESPrit3G speech processor with a directional microphone [Patrick et al., 2006] and the ACE or SPEAK speech-processing strategy with no autosensitivity or adaptive dynamic range optimization.

Bilateral Subjects. All 4 subjects (Bil1-Bil4) had a bilateral severe-profound sensorineural hearing loss that was assumed to be congenital. The mean age at first cochlear implantation was 1.9 years $(\mathrm{SD}=0.5)$, and none of the subjects consistently wore an HA in the non-implanted ear after implantation. The mean age at second cochlear implantation was 8.9 years $(\mathrm{SD}=2.4$ years), and all subjects had been full-time users of bilateral CIs for at least 12 months. The mean age at testing was 10.2 years $(\mathrm{SD}=2.4)$. All 4 subjects were using the ESPrit $3 \mathrm{G}$ speech processors in both ears with directional microphone and the ACE or SPEAK speech-processing strategy with no autosensitivity or adaptive dynamic range optimization. Details of the subjects' hearing loss, CIs and preoperative HAs are presented in table 3.

\section{Loudness Balancing}

The subjects were required to compare the loudness using either device alone after listening to a 1-min segment of a recorded story presented at $65 \mathrm{~dB}$ SPL. If the perceived loudness differed between the ears, then the HA or the 2 nd CI was adjusted to match the loudness of the first CI (1st CI). This was achieved by adjusting the volume control of the HA or applying a global C-level modifier to the speech processor map of the 2nd CI.

With the adjusted volume settings, the following steps were used to check the loudness balance between ears. In step 1, the subjects listened to the story presented at $59 \mathrm{~dB}$ SPL using the HA or the 2 nd CI alone, and then at $65 \mathrm{~dB}$ PL using the 1st CI. If loudness balancing had been achieved, the subject would be expected to report that the story presented to the HA or the 2nd CI was softer than that presented to the 1st CI. In step 2, the subjects then listened to the story presented at $65 \mathrm{dBSPL}$ using either device alone. Steps 1 and 2 were repeated until equal loudness was achieved at step 2 on at least 2 out of 3 trials.

Following this procedure, all except 1 subject achieved loudness balancing between the ears. For subject Bim8, the HA remained softer than the $\mathrm{CI}$ at the maximum volume control setting, and this setting was used for all testing. Four subjects (Bim1,
Bim9, Bil3, and Bil4) achieved loudness balancing without any adjustment to the HA or 2nd CI. Six bimodal subjects (Bim2, Bim3, Bim4, Bim5, Bim6, and Bim7) required an increase in HA gain of $6-11 \mathrm{~dB}($ mean $=8.2 \mathrm{~dB}, \mathrm{SD}=2.0)$. One bilateral subject (Bil1) required a global C-level increase in the 2 nd CI of $5 \%$, whilst another (Bil2) required a decrease of 3\%. Following loudness balancing, the possibility of saturation in the HAs with the adjusted volume was evaluated. SPL90 (the output sound pressure level for $90 \mathrm{~dB}$ SPL input) and gain values (at $65 \mathrm{~dB}$ SPL input) were obtained in a $2-\mathrm{cm}^{3}$ coupler for each HA. Saturation of the HA for the signal level used in this experiment ( $65 \mathrm{~dB}$ SPL) was possible only for subject Bim3, and the volume control was adjusted to a level just below the potential saturation point.

\section{Setup and Stimuli}

All testing was conducted in a sound-proof booth. Three Tannoy Reveal loudspeakers were positioned at ear level at a distance of $115 \mathrm{~cm}$ from the subject at $0^{\circ}$ (front), and at $90^{\circ}$ to the left and right. The stimulus was the Consonant-Nucleus-Consonant

Table 2. Aided hearing thresholds (dB SPL) in the non-implanted ear for the bimodal subjects

\begin{tabular}{llllll}
\hline Subject & $250 \mathrm{~Hz}$ & $500 \mathrm{~Hz}$ & $1 \mathrm{kHz}$ & $2 \mathrm{kHz}$ & $4 \mathrm{kHz}$ \\
\hline Bim1 & 55 & 45 & 45 & 60 & NR (80) \\
Bim2 & 55 & 45 & 40 & 55 & NR (80) \\
Bim3 & 40 & 35 & 40 & 60 & NR (80) \\
Bim4 & 45 & 40 & 40 & 40 & 70 \\
Bim5 & 55 & 55 & 50 & 70 & 75 \\
Bim6 & 50 & 50 & 65 & 60 & NR (80) \\
Bim7 & 45 & 45 & 55 & NR (80) & NR (80) \\
Bim8 & 45 & 45 & 40 & 60 & 60 \\
Bim9 & 55 & 50 & 45 & 65 & NR (80) \\
\hline
\end{tabular}

$\mathrm{NR}=$ No response at audiometer limits indicated in parentheses.

Table 3. Details of age, hearing loss, hearing aid usage prior to CIs, and CIs for bilaterally implanted subjects

\begin{tabular}{|c|c|c|c|c|c|c|c|}
\hline Subject & $\begin{array}{l}\text { Age } \\
\text { years }\end{array}$ & Etiology & $\begin{array}{l}\text { Age at bilateral } \\
\text { HA fitting, months }\end{array}$ & $\begin{array}{l}\text { HA usage before and } \\
\text { after } 1 \text { st } \mathrm{CI}^{1}\end{array}$ & $\begin{array}{l}\text { Age at 1st and } \\
2 \text { nd CI, years }\end{array}$ & $\begin{array}{l}\text { 1st and 2nd } \\
\text { CI types }\end{array}$ & $\begin{array}{l}\text { 1st and 2nd } \\
\text { CI strategies }\end{array}$ \\
\hline Bil1 & 12.8 & unknown & 11 & consistent/inconsistent & $1.8 / 11.3$ & CI22/CI24RCA & SPEAK \\
\hline Bil2 & 9.6 & unknown & 23 & inconsistent/inconsistent & $2.6 / 8.3$ & CI24M/CI24RCA & SPEAK \\
\hline Bil3 & 7.2 & unknown & 2 ; right ear only ${ }^{2}$ & consistent/none ${ }^{2}$ & $1.5 / 5.8$ & CI24M/CI24RCA & $\mathrm{ACE}$ \\
\hline Bil4 & 11.2 & unknown & 12 & consistent/inconsistent & $1.8 / 10.2$ & CI22/CI24RCA & SPEAK \\
\hline
\end{tabular}

CI22 = Straight-array implant with 20 electrodes plus 2 extracochlear electrodes; CI24RCA = soft-tip curved-array implant with 22 electrodes plus 2 extracochlear electrodes; SPEAK = spectral peak speech coding strategy; CI24M = straight-array implant with 22 electrodes plus 2 extracochlear electrodes; $\mathrm{ACE}=$ advanced combination encoder.

${ }^{1}$ Represents HA usage during the period between 1 st CI and 2 nd CI. ${ }^{2}$ Left ear not fitted due to atresia. 
(CNC) Word Test, presented at $65 \mathrm{~dB}$ SPL. The CNC words were phonemically balanced to Australian English [as used by Rance et al., 2007]. The noise was four-talker babble presented continuously at $55 \mathrm{~dB}$ SPL. This level was selected to provide a signal-tonoise ratio which limited floor and ceiling effects across the 3 device conditions.

\section{Evaluation Conditions}

Speech perception was evaluated in 3 device conditions and 2 noise conditions, for a total of 6 conditions. The device conditions for the bimodal subjects were $\mathrm{CI}$ alone (CI), HA alone (HA), and both devices together $(\mathrm{CI}+\mathrm{HA})$. The device conditions for the bilateral subjects were 1st CI alone, 2nd CI alone, and both CIs together $(\mathrm{CI}+\mathrm{CI})$. The 2 noise conditions were noise presented from the front, and noise presented from $90^{\circ}$ on the side of the CI (for bimodal subjects) or 1st CI (for bilateral subjects). Speech was presented from the front in both noise conditions. Four CNC word lists, a total of 200 words, were presented in each condition for each subject. The order of device and noise conditions was balanced within and across subjects over 4 test sessions.

\section{Procedure}

The subjects were instructed to repeat each word exactly as perceived, even for unknown words. The responses were recorded audiovisually using a Sharp Hi-8 analog camcorder with a plug-in microphone. The use of video recording enhances the accuracy of transcription by allowing transcription at a later time, repeat viewing, and inter- and intratranscriber consistency checking. Between device conditions, the subjects were engaged in conversation for a minimum of 2 min to allow adaptation to the new condition.

The responses were phonemically transcribed using a broad transcription method by a linguist experienced in transcribing the speech of children with a hearing impairment. All transcription was conducted in a sound-proof booth under headphones. The order of transcription was randomized across subjects. To counteract potential changes over time in the decision criteria, the linguist transcribed the first 12 lists of each subject and then transcribed the last 12 lists of each subject using the reverse order of subjects. For each transcribed list, the percentages of correctly identified words, phonemes, vowels, and consonants were calculated. A correct word score required that the correct phonemes were articulated in the correct order, with no additions or deletions. Correct order was not required for the phoneme, vowel, or consonant score. Thus, for example, for the stimulus word /pit/, a response of /tip/ or /pits/ was scored as 1 incorrect word, 3 correct phonemes, 1 correct vowel, and 2 correct consonants. To assess intratranscriber consistency, a randomly selected list from each subject was retranscribed without reference to the first transcription. To assess intertranscriber consistency, the first author independently transcribed 63 randomly selected lists.

The phonemic transcriptions were then entered into the INFOTRANSMIT program [Mok et al., 2006]. The program held in its dictionary the correct phonemic transcription of each $\mathrm{CNC}$ word obtained from The Macquarie Dictionary. INFOTRANS MIT compared the entered phonemic transcription with the relevant transcription in the dictionary and performed an information transmission analysis [Miller and Nicely, 1955]. A total of 9 information categories were analysed to cover the range of frequencies in speech [Mok et al., 2006]. These were sibilants $(/ \mathrm{J} /, / 3 /$, /dz/, /s/, /t $/, / \mathrm{z} /)$, fricatives $(/ \mathrm{e} /, / \mathrm{f} /, / \mathrm{z} /, / \mathrm{f} /, / \mathrm{h} /, / \mathrm{d} /, / \mathrm{s} /, / \mathrm{v} /, / \mathrm{z} /)$, bursts (/b/, /d/, /dz/, /t $/, / \mathrm{g} /, / \mathrm{k} /, / \mathrm{p} /, / \mathrm{t} /)$, plosives $(/ \mathrm{b} /, / \mathrm{p} /, / \mathrm{d} /, / \mathrm{t} /$, /g/, /k/), semivowels $(/ \mathrm{r} /, / \mathrm{w} /, / \mathrm{l} /, / \mathrm{j} /)$, nasals $(/ \mathrm{n} /, / \mathrm{m} /, / \mathrm{n} /)$, diphthongs (/ai/, /ei/, /or/, /av/, /ov/, /Iə/, / $/ \varepsilon \partial /)$, first formant $\left(\mathrm{F}_{1}\right)$, and second formant $\left(\mathrm{F}_{2}\right)$. The scores in the analyses of sibilants, fricatives, bursts, plosives, semivowels, nasals, and diphthongs represented the ability to perceive whether or not a phoneme belonged to the specific manner of articulation group. The scores in the analyses of $F_{1}$ and $F_{2}$ represented the ability to perceive whether a vowel had a high or low $\mathrm{F}_{1}$ (above or below $500 \mathrm{~Hz}$ ) and a high or low $\mathrm{F}_{2}$ (above or below $1500 \mathrm{~Hz}$ ), respectively. In the analyses of $F_{1}$ and $F_{2}$, diphthongs were excluded due to formant transitions.

\section{Results}

Using the method described in Shriberg and Lof [1991], the intratranscriber agreement was calculated as $97.8 \%$, and the intertranscriber agreement as $95.3 \%$.

\section{Performance in the Single-Ear Condition}

Figure 1 presents the mean percentage correct $\mathrm{CNC}$ phoneme scores for the individual subjects in the single ear conditions with noise from the front. For the bimodal subjects, paired $t$ tests indicated that CI scores were significantly greater than $\mathrm{HA}$-alone scores for each individual $(\mathrm{t} \geq 3.2, \mathrm{p} \leq 0.05$, mean difference $=30 \%)$. As performance with the $\mathrm{CI}$ was superior to that with the $\mathrm{HA}$, the CI was selected as the comparison condition when assessing bimodal advantage. For the bilateral subjects, paired $t$ tests indicated that scores with the 1st CI were significantly greater than those with the 2nd CI for 3 subjects ( $\mathrm{t} \geq 4.2, \mathrm{p} \leq 0.025$, mean difference $=31.8 \%$ ), but not for subject Bil4 $(\mathrm{t}=0.54, \mathrm{p}=0.6$, mean difference $=1.9 \%$ ). As performance with the 1 st CI was either superior to, or at least equal to, that with the 2 nd CI, the 1 st CI was selected as the comparison condition when assessing bilateral CI advantage.

To determine if the bimodal and bilateral groups differed in the HA or 2nd-CI scores in either noise condition, a two-way ANOVA was performed for each noise condition using subject group and CNC measure as factors, and HA or 2nd-CI scores as the dependent variable. Figure 1 presents only CNC phoneme scores in the HA and 2 nd-CI condition with noise from the front. In the noise front condition, results indicated a significant main effect for subject group and for CNC measure $(p<0.001)$. The interaction effect was not significant $(\mathrm{p}=0.15)$. Holm-Sidak pair-wise comparisons indicated that the bilateral group demonstrated higher scores with the 2 nd CI than did the bimodal group with the HA, with a mean 
Fig. 1. Mean $(n=4)$ percentage correct CNC phoneme scores for individual bimodal subjects (in the CI and HA conditions) and individual bilateral subjects (in the 1st-CI and 2nd-CI conditions) in the noise front condition. Error bars represent \pm 2 SEM.

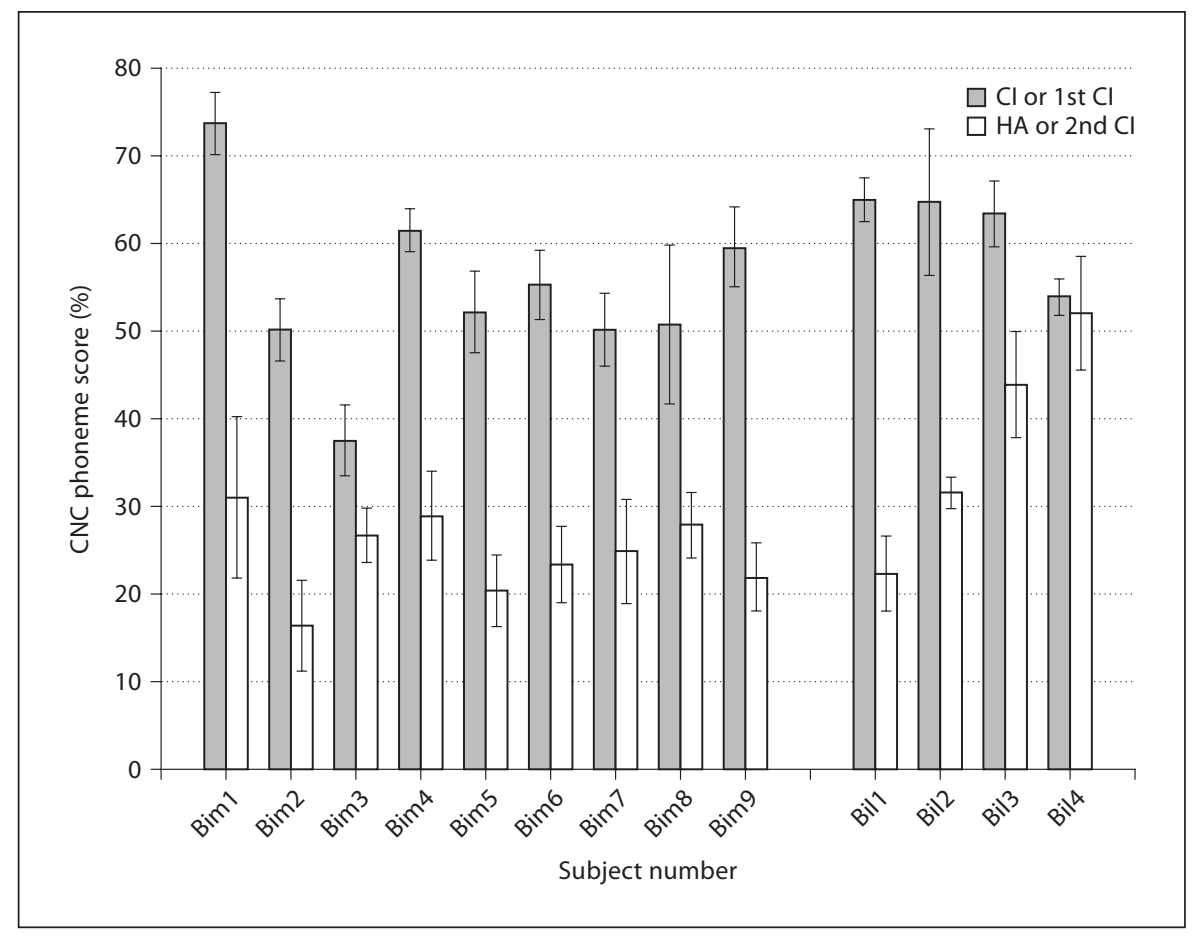

difference of $11.6 \%$ (95\% CI $=7.1-16.0$ ). In the noise CI/ 1st-CI condition, results again indicated a significant main effect for subject group and for CNC measure $(\mathrm{p}<$ $0.001)$, and a non-significant interaction effect ( $p=0.067)$. Holm-Sidak pair-wise comparisons indicated that the bilateral group demonstrated higher scores with the 2nd CI than did the bimodal group with the HA, with a mean difference of $17.1 \%(95 \% \mathrm{CI}=12.5-21.6 \%)$. These results indicate that the 2nd-CI-alone scores of the bilateral group were superior to the HA-alone scores of the bimodal group, irrespective of the noise direction or the $\mathrm{CNC}$ measure.

\section{Advantage Provided by the Second Device \\ Bimodal Subjects}

Figure 2 presents the mean percentage correct scores for the bimodal group, with noise from the front (fig. 2a) and from the side of the CI (fig. 2b). To determine if a significant bimodal advantage occurred, a two-tailed t test was used to compare the $\mathrm{CI}+\mathrm{HA}$ scores and the CI scores for each noise condition and each CNC measure (words, phonemes, consonants and vowels). In cases where the Kolmogorov-Smirnov test indicated data distribution was not normal ( $\mathrm{p}<0.05)$, a Mann-Whitney test was used. This applied to all $t$ tests reported in the Results section. Results showed a significant bimodal advantage for the group in both noise conditions for all $4 \mathrm{CNC}$ measures ( $\mathrm{p} \leq 0.01)$. In the noise front condition, the mean bimodal advantage was $6.3 \%$ (95\% CI $=1.6-10.9 \%)$. In the noise CI condition, the mean bimodal advantage was $8.1 \%(95 \% \mathrm{CI}=3.5-12.7 \%)$. These results show that, irrespective of the direction of the noise source, the group gained an advantage from using the HA in combination with their implant for the perception of words, phonemes, vowels and consonants.

To determine if a significant bimodal advantage occurred for individuals, a two-tailed $t$ test was used to compare the CI + HA scores and the CI scores for each subject. In the noise front condition, 6 out of 9 bimodal subjects demonstrated a significant $(\mathrm{p}<0.05)$ bimodal advantage on at least $1 \mathrm{CNC}$ measure (Bim2, Bim3, Bim4, Bim5, Bim6, and Bim8). In the noise CI condition, 8 out of 9 subjects (all except Bim4) demonstrated a bimodal advantage on at least $1 \mathrm{CNC}$ measure. For this group, it is important to remember that loudness was not balanced between the ears for 2 subjects (Bim 3 and Bim8); these subjects may have gained additional benefit if loudness was balanced. The results show that the majority of individual subjects gained an advantage from using the HA in combination with their implant for the perception of at least 1 of the words, phonemes, vowels or consonants. This occurred irrespective of the direction of the noise 
Fig. 2. Mean percentage correct scores for the bimodal group in the two noise conditions $(n=9)$. Significant differences between the $\mathrm{CI}+\mathrm{HA}$ and $\mathrm{CI}$ conditions are marked with an asterisk $(\mathrm{p}<0.05)$. Error bars represent \pm 2 SEM.

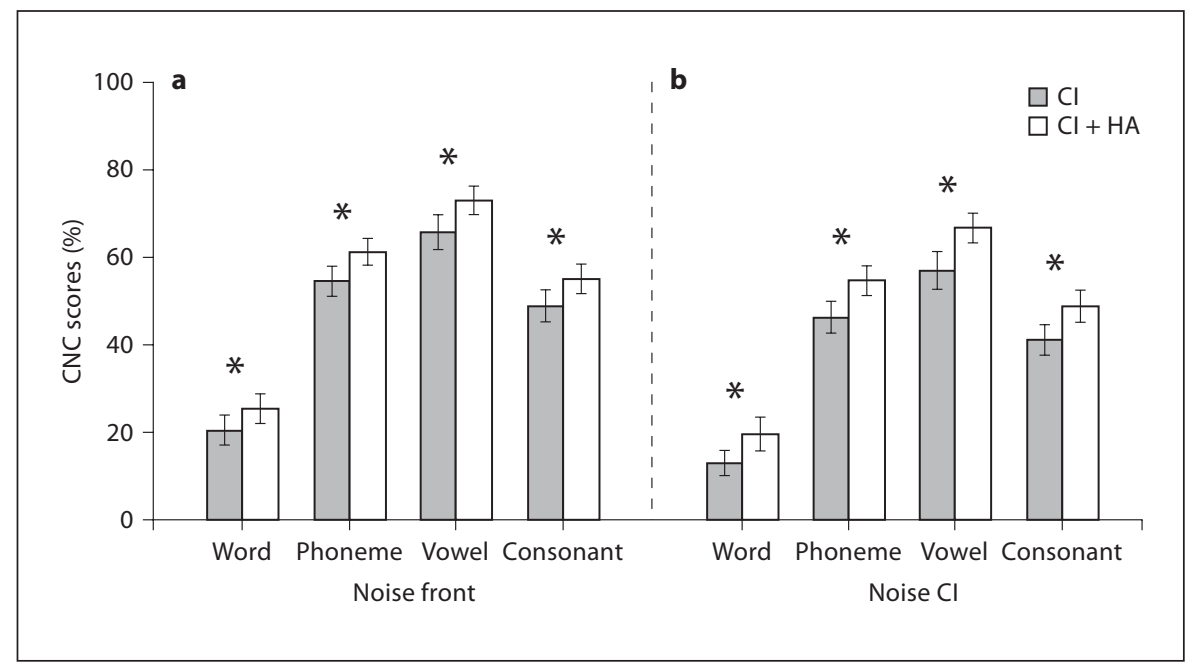

source. Furthermore, the results show that no subject demonstrated a disadvantage through additional use of an HA on any measure.

Pearson correlation analyses were used to examine the relationship between the bimodal advantage and aided thresholds at 250 and $500 \mathrm{~Hz}$ and 1 and $2 \mathrm{kHz}$ in the nonimplanted ear for the bimodal subjects. For subject Bim7, who had no aided response at $2 \mathrm{kHz}$ at the audiometer limit of $80 \mathrm{~dB}$ SPL, a threshold of $85 \mathrm{~dB}$ SPL was used. In the noise front condition, the correlations between the bimodal advantage and aided thresholds were not significant for any CNC measure ( $\mathrm{p}>0.05)$. In contrast, in the noise CI condition, significant negative correlations were found between aided thresholds at $500 \mathrm{~Hz}$ and the bimodal advantage for the perception of phonemes ( $\mathrm{r}=$ $-0.47, \mathrm{p}=0.004)$, vowels $(\mathrm{r}=-0.34, \mathrm{p}=0.041)$, and consonants $(r=-0.42, p=0.012)$. Significant negative correlations were also found between aided thresholds at 250 $\mathrm{Hz}$ and the bimodal advantage for the perception of phonemes $(\mathrm{r}=-0.35, \mathrm{p}=0.036)$. The correlations between bimodal advantage and the aided thresholds at 1 and $2 \mathrm{kHz}$ were not significant for any CNC measure ( $\mathrm{p}>$ $0.10)$.

To examine the relationship between bimodal advantage and aided threshold at $4 \mathrm{kHz}$, a two-tailed t test was used to compare the bimodal advantage for subjects with $4-\mathrm{kHz}$ aided thresholds of $\leq 75 \mathrm{~dB}$ SPL $(\mathrm{n}=3)$ and the subjects with no aided response at $4 \mathrm{kHz}(\mathrm{n}=6)$. In the noise front condition, there was no significant difference in bimodal advantage between the two groups. In the noise CI condition, the no-aided-response group demonstrated greater bimodal advantage in the perception of phonemes and consonants (the mean difference being $4.6 \%(\mathrm{t}=2.3, \mathrm{p}=0.028)$ and $4.5 \%(\mathrm{t}=2.1, \mathrm{p}=0.039)$, respectively). Overall, these results show a greater bimodal advantage for subjects with better aided thresholds at 250 and $500 \mathrm{~Hz}$, and subjects with poorer aided thresholds at $4 \mathrm{kHz}$ (i.e., threshold greater than $75 \mathrm{~dB} \mathrm{SPL}$ ).

\section{Bilateral Subjects}

Figure 3 presents the mean percentage correct scores for the bilateral group, with noise from the front (fig. 3a) and from the side of the 1st CI (fig. 3b). To determine if a significant bilateral CI advantage occurred, a two-tailed $\mathrm{t}$ test was used to compare the CI + CI scores and the 1st$\mathrm{CI}$ scores for each noise condition and each CNC measure (words, phonemes, consonants and vowels). In the noise front condition, there was no significant bilateral CI advantage for any of the $4 \mathrm{CNC}$ measures ( $\mathrm{p}>0.10$ ). In contrast, in the noise 1st-CI condition, results showed a significant bilateral CI advantage for all $4 \mathrm{CNC}$ measures $(\mathrm{p}<0.05)$, with a mean advantage of $7.0 \%(95 \% \mathrm{CI}=0.01-$ $14.0 \%)$. These results show that, when noise was presented from the side of the 1st CI, the group gained an advantage from using the 2nd CI in combination with the 1st for the perception of words, phonemes, vowels and consonants. When the noise was presented from the front, no such advantage was gained, however; nor was there any disadvantage through use of the 2nd CI.

To determine if a significant bilateral CI advantage occurred for individuals, a two-tailed t test was used to compare the CI + CI scores and the 1st-CI scores for each subject. In the noise front condition, a bilateral CI advantage was demonstrated for only 1 subject on 1 measure, 
Fig. 3. Mean percentage correct scores for the bilateral group in the two noise conditions $(n=4)$. Significant differences between the $\mathrm{CI}+\mathrm{CI}$ and CI conditions are marked with an asterisk $(\mathrm{p}<0.05)$. Error bars represent \pm 2 SEM.

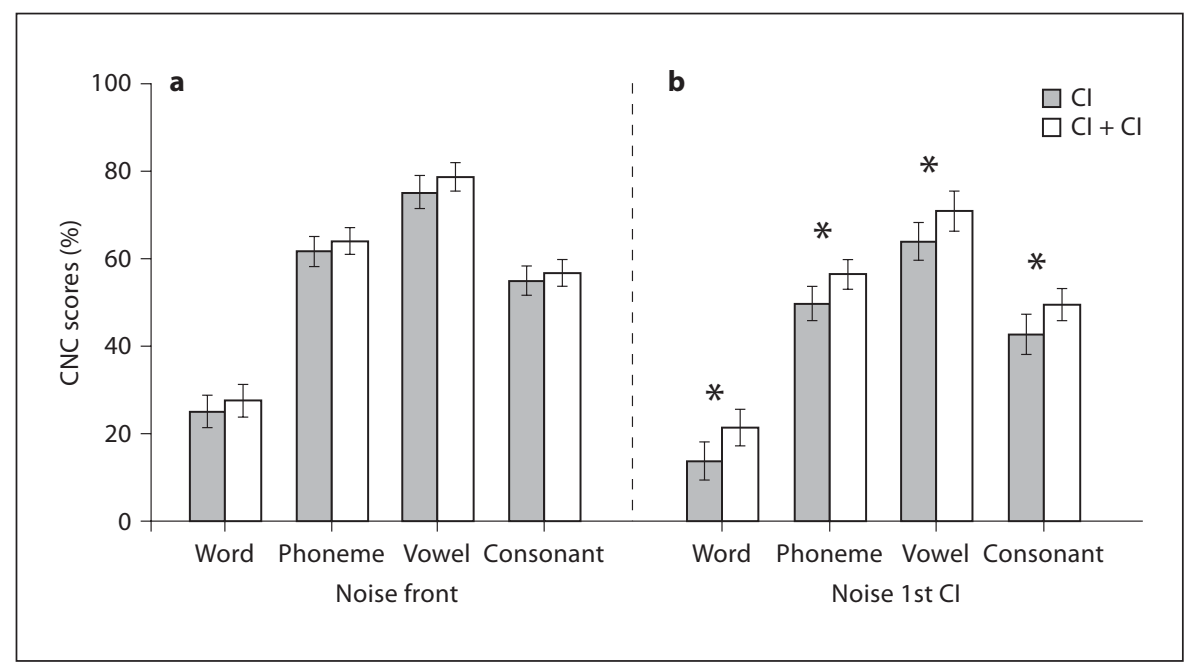

this being for Bil4 on phoneme perception ( $p=0.044)$. In the noise 1st-CI condition, 2 out of the 4 subjects (Bil2 and Bil3) demonstrated a bilateral CI advantage in at least $1 \mathrm{CNC}$ measure $(\mathrm{p}<0.05)$. These results show that the number of individuals demonstrating an advantage through use of the 2nd CI was limited to 1 (with noise from the front) or 2 (with noise from the side of the 1st $\mathrm{CI}$ ) subjects on at least 1 (and up to a maximum of 3) CNC measures. However, the results also show that no subject demonstrated a disadvantage through additional use of the 2 nd CI.

Bimodal versus Bilateral Subjects

To determine if the bimodal and bilateral groups differed in the advantage provided by the second device, a two-way ANOVA was performed for each noise condition using subject group and CNC measure as factors, and bimodal or bilateral CI advantage as the dependent variable. Bimodal advantage was calculated as $\mathrm{CI}+\mathrm{HA}$ scores - CI scores, and bilateral CI advantage as CI + CI scores - 1st-CI scores for the bilateral subjects. In the noise $\mathrm{CI} / 1$ st-CI condition, results indicated no significant main effect for subject group $(p=0.39)$ or CNC measure $(\mathrm{p}=0.89)$. In the noise front condition, results indicated a significant main effect for subject group ( $\mathrm{p}=$ 0.007 ) but no significant main effect for CNC measure $(\mathrm{p}=0.83)$ and no significant interaction effect $(\mathrm{p}=0.95)$. Holm-Sidak pair-wise comparisons indicated that the bimodal advantage demonstrated by the bimodal group was greater than the bilateral CI advantage demonstrated by the bilateral group, with a mean difference of 3.7\% $(95 \% \mathrm{CI}=1.0-6.3 \%)$. These results suggest that, when noise was presented from the side, there was no difference in the degree of advantage gained through use of the HA versus the 2 nd $\mathrm{CI}$ for any $\mathrm{CNC}$ measure. However, when noise was presented from in front, the bimodal group gained more advantage from the $\mathrm{HA}$ than the bilateral group gained from the 2 nd $\mathrm{CI}$ for all CNC measures.

The advantage provided by the second device for information transmitted within each phoneme category was calculated and compared across the two subject groups. Figures 4 and 5 present the mean bimodal advantage for the bimodal group and the mean bilateral CI advantage for the bilateral group for the noise front condition and the noise CI/1st-CI condition, respectively. The advantage for 9 phoneme categories is presented, with high-frequency groups on the left and low-frequency groups on the right. Results obtained for adults with CNC words presented in quiet [Mok et al., 2006] are included in figure 5 for comparison. In the noise front condition (fig. 4), there was a bimodal advantage for the bimodal group for all phoneme categories except sibilants. In contrast, for the bilateral group, there was a bilateral CI advantage for only 5 phoneme categories. The bimodal group demonstrated greater advantage than the bilateral group for most phoneme categories. In the noise CI/1stCI condition (fig. 5), there was a bimodal advantage for the bimodal group and a bilateral CI advantage for the bilateral group for all phoneme categories, with the degree of advantage generally greater than that in the noise front condition. Similar to the noise front condition, the bimodal group demonstrated greater advantage than the bilateral group for most phoneme categories. In summary, these results show an almost consistent pattern across 
Fig. 4. Mean bimodal and bilateral CI advantage in information transmission for the bimodal and bilateral groups, respectively, in the noise front condition.
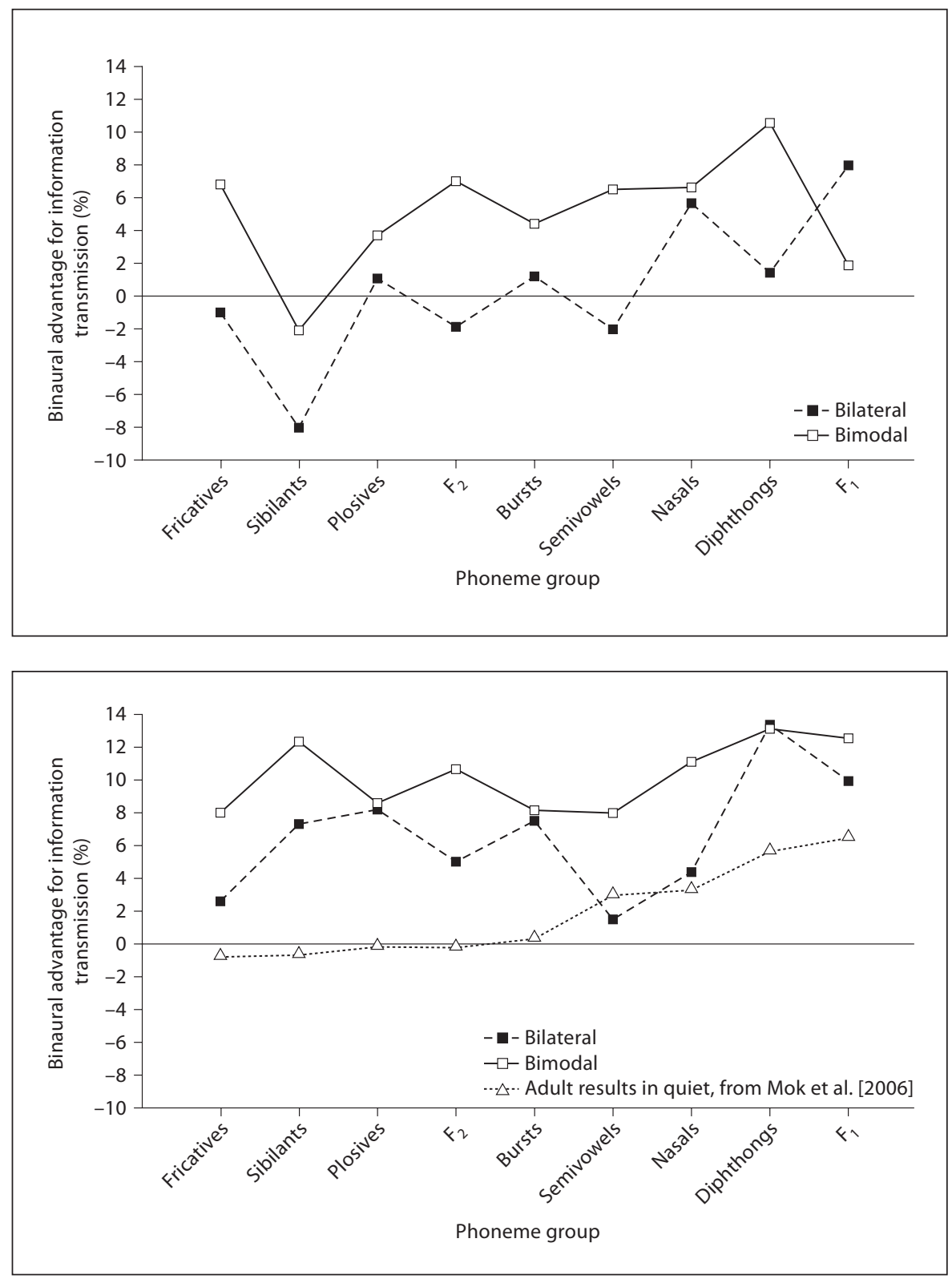

Fig. 5. Mean bimodal and bilateral CI advantage in information transmission for the bimodal and bilateral groups, respectively, in the noise CI/1st-CI condition. Results from adults from Mok et al. [2006] are included for comparison. phoneme categories of greater advantage from the second device for both the bimodal and bilateral groups when noise was presented from the side compared with the front, and generally a greater advantage for the bimodal group than for the bilateral group, irrespective of the direction of the noise source.

\section{Headshadow Advantage}

If a headshadow advantage was gained through the use of a second device, a greater bimodal or bilateral CI advantage would be expected in the noise CI/1st-CI con- dition as compared with the noise front condition. To determine if this did occur, a two-way ANOVA was performed for each subject group using noise condition and CNC measure as factors, and bimodal or bilateral CI advantage as the dependent variable. For the bimodal subjects, results indicated no significant main effect for noise condition or CNC measure ( $\mathrm{p}=0.069$ and $\mathrm{p}=0.27$, respectively). This result suggests that the bimodal subjects were not gaining headshadow advantage through use of the additional HA. For the bilateral subjects, results indicated a significant main effect for noise condition $(\mathrm{p}=$ 
$0.009)$, but not for CNC measure $(\mathrm{p}=0.97)$. The interaction effect was not significant $(\mathrm{p}=1.0)$. Holm-Sidak pairwise comparisons indicated that greater bilateral CI advantage occurred in the noise 1st-CI condition than the noise front condition, with a mean difference of $4.4 \%$ $(95 \% \mathrm{CI}=1.2-7.6 \%)$. This result suggests that the bilateral subjects were gaining headshadow advantage through use of the additional 2nd CI, and that this advantage was gained for the perception of words, phonemes, consonants, and vowels.

\section{Discussion}

\section{Binaural Advantage}

Most of the bimodal and bilateral subjects (all except Bill) in this study demonstrated significant improvement in speech perception in at least 1 noise condition when wearing binaural devices compared to a single CI. Together with the findings of previous studies [see, for example, Ching et al., 2001; Mok et al., 2006; Galvin et al., 2007], these results reinforce the importance of fitting a second device to children who use a CI. For the bilateral subjects, these results suggest that lack of consistent HA use or a long time gap between the two implants does not preclude speech perception advantage from a 2 nd CI. It is possible, however, that more HA usage prior to receiving the 2 nd $\mathrm{CI}$ or a shorter time gap between the implants could lead to a greater bilateral CI advantage for these subjects.

An important point related to the calculation of bimodal and bilateral CI advantage should be noted. As performance was significantly superior in the CI/1st-CI condition, the ear with the CI/1st-CI condition was selected as the 'better ear' at the time of testing and used as the comparison condition when assessing the advantage provided by the second device. This ear may not have been the 'better ear' in terms of hearing thresholds prior to the first implantation, however it was the 'better ear' in terms of speech perception at the time of testing. Only a prospective study recruiting subjects prior to their first implantation could attempt to compare performance in the bimodal or bilateral condition with unilateral performance using the original 'better ear' (i.e., the ear with superior hearing thresholds pre-operatively). Even such a prospective study would be limited, as the majority of children receive a first implant in their poorer ear. The advantage of the present study is that it is more similar to the majority of real-life choices between an HA or second implant, where the first implanted ear was originally the 'poorer ear' (based on hearing thresholds or cochlear scans) but has become the 'better ear' because it has been the ear receiving better quality acoustic input from a young age.

\section{Aided Thresholds and Bimodal Advantage}

The results of the correlation analyses showed that the bimodal subjects with better aided thresholds at low frequencies (250 and $500 \mathrm{~Hz}$ ) demonstrated greater bimodal advantage. This is possibly due to the fact that an HA provides finer low-frequency spectral information than a CI, so this information could be complementary to the signal received from an implant in the opposite ear. A previous study also showed that adult implant users could utilize the low-frequency acoustic information from a contralateral HA to improve the perception of low-frequency phonemes [Mok et al., 2006].

The results of the present study also indicate that the bimodal subjects with poorer aided thresholds at $4 \mathrm{kHz}$ demonstrate greater bimodal advantage than those with better $4-\mathrm{kHz}$ thresholds. This is similar to the results for adults, which also indicate that subjects with poorer aided thresholds at $4 \mathrm{kHz}$ demonstrate greater bimodal advantage [Mok et al., 2006]. A possible explanation is that there may be a mismatch of high-frequency information provided by the HA and the CI. This could be due to the different places of excitation in the cochleae resulting from electric versus acoustic stimulation [Blamey et al., 1996], and may limit the bimodal benefit gained. It is also possible that, given that the NAL-RP formula was used (which provides amplification in the high frequencies) and that the subjects were all profoundly hearing impaired, dead regions in the cochlea were being stimulated [Moore, 2001]. If so, and off-frequency listening was occurring, there may have been a masking effect which limited the bimodal benefit that was gained by subjects with better aided hearing at $4 \mathrm{kHz}$. These results raise the possibility that a child may gain greater bimodal benefit if the high frequencies alone are stimulated by the CI and only low-frequency information is provided by the HA. However, research with bimodal adults has provided no evidence that this combination is likely to increase bimodal benefit [Simpson, 2007]. A particular disadvantage of such an approach would be the reduction in the amount of information provided by the CI, which is, in the majority of cases, the superior and preferred listening ear. A possible alternative would be limiting the frequency range of the HA only.

The present group showed no correlation between aided thresholds in the mid-frequencies $(1$ and $2 \mathrm{kHz})$ and 
bimodal advantage. This result differs from that found for adults [Mok et al., 2006], who demonstrated a significant correlation between poorer aided mid-frequency thresholds and greater bimodal benefit. If, as suggested above, the mismatch of information between the ears does exist for bimodal users, it may be less significant in children due to the use of bimodal input from a young age.

Overall, these results suggest that individual variability in bimodal outcome could be partly accounted for by the differences in aided thresholds, and that better aided thresholds do not necessarily result in greater bimodal advantage.

\section{Bimodal versus Bilateral Subjects}

Noise Front Condition

In the noise front condition, where a headshadow effect was absent, the bimodal subjects demonstrated a significant bimodal advantage. This result suggests that the bimodal subjects were able to combine the acoustic and electric signals from the two ears to improve speech perception. In contrast, the bilateral subjects did not demonstrate a significant bilateral CI advantage in the noise front condition. It is possible that binaural processes such as binaural redundancy are minimal in the bilateral subjects. Since the number of bilateral subjects in this study is small $(\mathrm{n}=4)$, it is possible that the small bilateral CI advantage observed in the noise front condition would be statistically significant with a greater number of subjects. The fact that, in the noise front condition, the bimodal advantage shown was significantly greater than the bilateral CI advantage is in agreement with the results of an earlier study assessing speech detection in noise [Mok et al., 2007]. This is possibly due to the fact that an HA is able to provide finer low-frequency spectral information than a CI, and hence, can add information about speech that the CI does not convey well [Mok et al., 2006].

\section{Information Transmission Analyses}

In the noise CI condition, the bimodal subjects demonstrated more bimodal advantage in the perception of diphthongs and $F_{1}$ than other phoneme groups. This is similar to a previous study in which bimodal adults also showed the most bimodal advantage for these two phoneme groups [Mok et al., 2006] (see fig. 5). Unlike the adults, however, the present subjects also demonstrated a relatively large bimodal advantage for some of the other phoneme groups, including those that require perception of higher-frequency information (e.g. fricatives, sibilants, $\mathrm{F}_{2}$ ). Another unexpected result was that the information transmission analyses did not show a clearly distinguishable difference in the pattern of bimodal or bilateral CI advantage across phoneme groups. As mentioned in the Introduction, results for adults have led to the hypothesis that the bimodal subjects would demonstrate more bimodal advantage for the lower-frequency phoneme groups whilst the bilateral subjects would demonstrate more bilateral CI advantage for a wider range of frequencies or mostly in the high frequencies. There could be a few factors contributing to the unexpected results. Firstly, the bimodal adults were tested in quiet whilst the present bimodal subjects were tested in noise. It is possible that the perception of higher frequencies is more important when listening in noise. Secondly, the bimodal children had been using the bimodal input from a young age, while the bimodal adults only received the CI in adulthood. Hence, as also suggested by the aided-threshold results, it is possible that the children have learnt to better integrate the signals from the two ears, and that the mismatch between the ears at higher frequencies is less significant for children.

\section{Headshadow Advantage}

In addition to the mechanisms for bimodal or bilateral CI advantage in the noise front condition, headshadow advantage and binaural unmasking could also contribute to the advantage shown in the noise CI/1st-CI condition. Past studies have shown that binaural unmasking in bimodal and bilateral subjects is absent or very minimal relative to headshadow advantage [see, for example, van Hoesel and Tyler, 2003; Ching et al., 2005]. Thus, the difference in the advantage shown in the noise front as compared with the noise CI/1st-CI conditions could give an estimate of headshadow advantage, that is, the advantage of having the HA or the 2nd CI 'shadowed' from the noise.

The bilateral subjects demonstrated a significantly greater bilateral CI advantage in the noise $\mathrm{CI} / 1$ st-CI condition than the noise front condition, while the bimodal subjects demonstrated no significant difference in bimodal advantage between the 2 noise conditions. These results suggest that headshadow advantage was present for the bilateral subjects but not for the bimodal subjects in the noise CI/1st-CI condition. This is similar to a previous pediatric study which also showed that the 2nd CI provides greater headshadow advantage than the HA [Mok et al., 2007]. A combination of 2 factors may have contributed to the difference in headshadow advantage between a 2nd CI and an HA. Firstly, the headshadow effect improves the signal-to-noise ratio mainly in the high 
frequencies. The fact that the subjects' HA provided limited or no access to high-frequency sounds (evidenced by the aided thresholds) may have limited the headshadow advantage for the bimodal subjects. Secondly, the bilateral subjects had better speech perception with the 2 nd CI than the bimodal subjects had with the HA. This is despite the fact that the bilateral subjects had less experience with the 2nd CI than the bimodal subjects had with the HA. Hence, when the noise was on the side of the 1st $\mathrm{CI}$, the bilateral subjects could shift attention to the 2 nd $\mathrm{CI}$ and benefit from the improved signal-to-noise ratio at the 2 nd CI. In contrast, the bimodal subjects may not have benefited from such a shift of attention as the signal from the HA was very poor relative to the CI, despite the better signal-to-noise ratio at that ear. This suggests that, for a child with a severe-to-profound hearing loss and minimal access to the speech spectrum at high frequencies, there is a greater speech perception potential with a 2nd CI than an HA alone.

It is important to remember that the evaluation conditions in the present study involved speech from the front only; in real life, speech is often presented from other directions, particularly from the side during group discussions such as occur in meetings or in the classroom. Therefore, the superior speech perception potential of a 2nd CI will provide additional functional benefit over an $\mathrm{HA}$, as the HA wearer may need to orientate his or her better (implanted) ear to the speaker on one side in order to achieve adequate perception. Further functional benefit is achieved with the potential for the 2nd CI to act as a back-up to the 1st CI at times when batteries go flat or hardware breaks down, or even more importantly, reimplantation of one ear is required. It is also necessary to remember that direct comparisons of the bilateral and bimodal groups are limited by the fact that there was no matching of subjects and the two groups differed in important characteristics, such as age at implantation and hearing level. The average age at which the first or only CI was received was 6.8 years $(\mathrm{SD}=2.6)$ and 1.9 years $(\mathrm{SD}=0.5)$ for the bimodal and bilateral groups, respectively. Nor were the groups equivalent in terms of hearing level in the second ear (i.e., the HA or 2nd CI ear). The bimodal group, by definition, consisted of subjects with sufficient residual hearing in the second ear to make HA use worthwhile. In contrast, subject Bil3 was unable to wear a HA (due to atresia), and the remaining 3 bilateral subjects refused to wear an HA consistently due to lack of perceived benefit (all had unaided thresholds $\geq 100 \mathrm{~dB}$ $\mathrm{HL}$ from $250 \mathrm{~Hz}$ to $4 \mathrm{kHz}$ ). How such differences between the groups may have affected the results cannot be pre- dicted. It will always be very difficult to match bimodal and bilateral CI subjects for hearing loss, given that it is children with more residual hearing who are more likely to have a bimodal fitting and children with less residual hearing who are more likely to use a single implant alone or receive a 2 nd CI.

Overall, these results suggest that, for children with implants, an HA could provide greater additional advantage than a 2nd CI for speech perception in noise when speech arrives from in front; however, this requires that the child has some useful residual hearing in the second ear and is willing to wear an HA. In contrast, a 2nd CI is likely to provide greater additional benefit when speech is presented from the side, and greater speech perception benefit when used alone. The mechanisms underlying the advantage from the second device probably differ in the bimodal and bilateral groups. For the bimodal group, the additional speech information contained in the acoustic signal may be the major source of bimodal advantage. For the bilateral group, the headshadow effect may provide the major advantage. As the number of bilateral subjects was very small in this study, results should be interpreted with caution and further studies involving a greater number of subjects would be important. Despite the small number of bilateral subjects, however, the results are similar to other studies showing a headshadow effect as the main contribution to the bilateral CI advantage [see, for example, van Hoesel and Tyler, 2003; Galvin et al., 2007]. Future studies comparing other aspects of potential additional benefit from an HA or a 2nd CI, such as cost-benefit analyses and benefits to localization, would be helpful to refine clinical selection criteria for a 2nd CI. Further comparisons involving other subject populations would also be of interest such as bilateral subjects with simultaneous CIs or receiving the $2 \mathrm{nd} \mathrm{CI}$ at a younger age or greater experience with the $2 \mathrm{nd} \mathrm{CI}$, and bimodal subjects with a lesser degree of residual hearing. It is possible that the amount of advantage provided by the second device would be different in these populations.

\section{Conclusion}

Significant improvement in speech perception was demonstrated by most subjects in at least 1 noise condition when wearing binaural devices compared to a single CI. Results suggested that, for children able and willing to use an HA, an HA could provide greater advantage than a second implant for speech perception in noise, at 
least when speech and noise are presented from in front. In contrast, a 2 nd CI could provide greater speech perception than an HA when used by itself, or when speech was presented on the side of the 2 nd CI. The mechanisms underlying the advantage provided by the second device may be different in the bimodal and bilateral groups. For the bimodal group, the ability to combine the additional speech information contained in the acoustic signal with the electric signal may be the major source of advantage. For the bilateral group, the headshadow effect may provide the major advantage. Information transmission analysis did not show a clear difference between the subject groups in the pattern of advantage provided by the second device across phoneme groups. For the bimodal subjects, those with better aided thresholds at 250 and $500 \mathrm{~Hz}$, and/or poorer aided thresholds at $4 \mathrm{kHz}$ demonstrated greater bimodal advantage. A greater number of subjects will be required in future studies to verify the applicability of these findings to the wider population of pediatric CI users. These findings, however, encourage the use of bimodal devices and bilateral implants in children, give insight into the individual variability in bimodal outcome, and enhance understanding of the differences between an HA and a second implant when used together with an implant in the opposite ear.

\section{Acknowledgements}

We greatly appreciate the time and effort of the children and their family members for participating in the study. We would also like to thank Dr. Shani Dettman for helping in the recruitment of subjects, Dr. David Grayden for the software designs, Dr. Richard van Hoesel for the valuable input, Colleen Holt for transcribing the responses, and two anonymous reviewers for their constructive comments.

\section{References}

Blamey PJ, Dooley GJ, Parisi ES, Clark GM: Pitch comparisons of acoustically and electrically evoked auditory sensations. Hear Res 1996; 99:139-150.

Ching TY, Incerti P, Hill M: Binaural benefits for adults who use hearing aids and cochlear implants in opposite ears. Ear Hear 2004;25: $9-21$.

-Ching TY, Psarros C, Hill M, Dillon H, Incerti P: Should children who use cochlear implants wear hearing aids in the opposite ear? Ear Hear 2001;22:365-380.

-Ching TY, van Wanrooy E, Hill M, Dillon $\mathrm{H}$ Binaural redundancy and inter-aural time difference cues for patients wearing a cochlear implant and a HA in opposite ears. Int $\mathrm{J}$ Audiol 2005;44:513-521.

Dunn CC, Tyler RS, Witt SA: Benefit of wearing a hearing aid on the unimplanted ear in adult users of a cochlear implant. J Speech Lang Hear Res 2005;48:668-680.

Galvin KL, Leigh JR, Hughes KC: How we do it: clinical management of the child receiving a second, bilateral cochlear implant. Cochlear Implants Int, in press.
Galvin KL, Mok M, Dowell RC: Perceptual benefit and functional outcomes for children using sequential bilateral cochlear implants. Ear Hear 2007;28:470-482.

Kong YY, Stickney GS, Zeng FG: Speech and melody recognition in binaurally combined acoustic and electric hearing. J Acoust Soc Am 2005;117(3 pt 1):1351-1361.

Litovsky RY, Johnstone PM, Godar S: Benefits of bilateral cochlear implants and/or hearing aids in children. Int J Audiol 2006a;45(suppl 1):S78-S91.

Litovsky RY, Johnstone, PM, Godar S, Agrawal S, Parkinson A, Peters R, Lake J: Bilateral cochlear implants in children: localization acuity measured with minimum audible angle. Ear Hear 2006b;27:43-59.

Mok M, Galvin KL, Dowell RC, McKay CM: Spatial unmasking and binaural advantage for speech detection in children with normal hearing, bimodal devices and bilateral cochlear implants. Audiology Neurootol 2007; 12:19-30.

Mok M, Grayden D, Dowell R, Lawrence D: Speech perception for adults who use hearing aids in conjunction with cochlear implants in opposite ears. J Speech Lang Hear Res 2006;49:338-351.
Moore BCJ: Dead regions in the cochlea: diagnosis, perceptual consequences, and implications for the fitting of hearing aids. Trends Amplif 2001;5:1-34.

Patrick JF, Busby PA, Gibson PJ: The development of the Nucleus ${ }^{\circledR}$ Freedom ${ }^{\mathrm{TM}}$ cochlear implant system. Trends Amplif 2006;10: $175-200$.

Rance G, Barker E, Mok M, Dowell R, Rincon A, Garratt R: Speech perception in noise for children with auditory neuropathy/dyssynchrony type hearing loss. Ear Hear 2007;28: 351-360.

-Shriberg L, Lof G: Reliability studies in broad and narrow phonetic transcription. Clin Linguist Phon 1991;5:225-279.

Simpson A: Improving high-frequency audibility for hearing-impaired listeners using a cochlear implant or frequency-compression aid; unpublished doct. thesis, Melbourne, 2007.

van Hoesel RJ, Tyler RS: Speech perception, localization, and lateralization with bilateral cochlear implants. J Acoust Soc Am 2003; 113:1617-1630. 